\title{
Determinação da sensibilidade de transdutores ultrassônicos para fisioterapia pelo método da autorreciprocidade
}

\author{
Viviane Mendes Abrunhosa*, Rodrigo Pereira Barretto da Costa-Félix, Marco Antônio von Kruger, \\ Wagner Coelho de Albuquerque Pereira
}

\begin{abstract}
Resumo A radiação ultrassônica é frequentemente utilizada em fisioterapia para tratar lesões de tecidos moles. Entretanto, os profissionais de fisioterapia não têm como saber, com exatidão, a quantidade de energia emitida pelos transdutores destes equipamentos caso a calibração dos mesmos não seja apropriadamente realizada. $\mathrm{O}$ objetivo do presente estudo é mostrar uma forma de calibrar a sensibilidade de transmissão de transdutores ultrassônicos de fisioterapia pelo método de autorreciprocidade. A título de exemplo, este método foi testado na calibração de três transdutores, os resultados obtidos com os coeficientes de variação foram inferiores a $6 \%$. Observou-se que as sensibilidades de 1 dos 3 transdutores avaliados é linear, dentro da faixa da frequência utilizada para uma tensão de excitação variando de 3,5 a 10,5 V medidos pico a pico, enquanto os outros 2 não são lineares nesta mesma faixa de tensões. Isto indica que pode haver casos em que o aumento do sinal de excitação não implique o correspondente aumento proporcional da energia ultrassônica. Esta informação precisa ser levada em consideração quando executado o projeto da eletrônica do equipamento, a fim de assegurar que a escala nominal seja fiel à potência acústica gerada. Os procedimentos experimentais foram baseados na normalização internacional de calibração de hidrofones (transdutores).
\end{abstract}

Palavras-chave Metrologia, Ultrassom, Calibração, Transdutor, Fisioterapia.

\section{Sensitivity determination of physiotherapy ultrasonic transducers by the self-reciprocity method}

\footnotetext{
Abstract Ultrasonic irradiation is frequently used in physiotherapy to treat soft tissue injuries. It must be pointed however that physiotherapists have no means to know exactly the amount of energy emitted from the transducers of such equipments because of limitations in their calibration. The objective of the present study is to show one possible way to perform sensitivity calibration of ultrasonic physiotherapy transducers by a self-reciprocity method. As exemplification the method was tested in the calibration of 3 transducers for an excitation voltage varying from 3.5 to $10.5 \mathrm{Vpp}$ and the results show a relative error of less than $6 \%$. It was observed that, on their respective frequency band, one transducer was linear and two were not linear, for the same excitation voltage. This indicates that for some transducers a linear increase in excitation voltage may not correspond to a proportional increase in the ultrasonic output energy. Such information must be taken into account in the electronics project of the equipment in order to ensure that the nominal scale is a reliable indicator of the acoustic power generated. The experimental procedures were based on international standards (IEC 60866). The following step is to adapt this method to characterize ultrasound beams with exciting tensions capable to generate acoustic intensities in therapeutic levels.
}

Keywords Metrology, Ultrasound, Calibration, Transducers, Physiotherapy. 


\section{Extended Abstract}

\section{Introduction}

The ultrasonic wave is a disturbance mechanical frequency above $20 \mathrm{kHz}$. In medicine, ultrasound (US) is applied both to investigate the properties of the tissue (seeking a diagnosis) and to modify these properties (therapy). Using this feature, as a form of treatment aims to stimulate the natural process of tissue healing and can be applied in different types of injuries. Currently, the method routinely used by professionals to ensure that equipment is emitting ultrasonic radiation is only visual. The absence of metrology as a supporting element, incorporating the daily lives of practitioners, can generate a lack of credibility of the implementation of this therapeutic resource (Costa-Félix and Azevedo, 2003). The method used in this study to characterize the transducers is the calibration self-reciprocity, originally described in International Electrotechnical Commission 60866 standard (IEC, 1987), which aims to determine the sensitivity of the transducer. This standard has been canceled and replaced by International Electrotechnical Commission 62127-2 (IEC, 2007). However, the standard reference in this work will be the IEC 60866, because the more recent version does not detail the procedure employed.

The aim of this study is to implement and validate the calibration method for self-reciprocity to characterize the sensitivity of ultrasonic transducers used in physical therapy and highlight the contribution of metrology as a tool to support reliability in this type of technology in health.

\section{Materials and Methods}

Measurements were undertaken in an acrylic walled water bath, and a stainless steel cylinder as reflecting target. Voltages were measured with an oscilloscope (National Instruments, model NI PXI 5112) and the ultrasound signal was generated with an arbitrary function generator (National Instruments, model NI PXI 5442). Current was calculated through the voltage difference in an inline $56 \Omega$ nominal resistor, which was calibrated with the aid of a vector impedance meter (Agilent Technologies, model 4294A). All measurements were automatically performed using a homemade automation system developed in LabVIE $W^{\circledR}$ platform. Devices under test were one ultrasonic transducer manufactured by Panametrics-NDT, model A304S (Ø $=25.4 \mathrm{~mm}$, nominal frequency of $2.25 \mathrm{MHz})$ and two physiotherapy ultrasonic transducers (nominal frequency of $1.0 \mathrm{MHz}$ ), belonging to the Laboratory of Ultrasound of the Biomedical Engineering Program (PEB) of COPPE/UFRJ.
Five measurements were performed in repeatability conditions. In each measurement, driven voltage ranged from 3.5 to $10.5 \mathrm{~V}$, measured peak to peak, in order to evaluate device linearity. Excitations consisted in stepped sine tone-burst. Uncertainties were assessed for each frequency, each measurement and for each driven voltage, and its budged was defined accordingly to ISO-GUM (2008) requirements. For each frequency, sensitivity was considered validated if linearity was less than $10 \%$ and combined uncertainty was less than $9 \%$. Besides, measurements performed with the reference standard should not be larger than \pm twice the combined uncertainty. The system was considered valid to perform calibration just in those frequencies were all validation criteria applied. The system diagram is shown in Figure 1.

\section{Results}

According to the defined linearity criteria, one of the $1.0 \mathrm{MHz}$ ultrasonic transducers calibrations were not considered valid from 0.9 to $1.15 \mathrm{MHz}$ and the other was considered valid from 1.05 to 1.1 MHz for uncertainty criteria, as disclose in Table 2 . The 2.25 MHz ultrasonic transducers were considered valid from 1.5 to $2.7 \mathrm{MHz}$ for uncertainty criteria, as disclose in Table 2 . Figures 10 to 12 present results of linearity for the three ultrasonic transducers, respectively. Those figures show the range of sensitivity with increase of voltage in different frequency.

\section{Discussion and Conclusion}

Although not a requirement of the Agência de Vigilância Sanitária - ANVISA, sensitivity is one of the parameters that have an important role both in performance as well as optimization of the manufacturing of appliances and their transducers.

This study presents an implementation of the calibration by self-reciprocity according to the standard International Electrotechnical Commission 60866 (IEC, 1987), applied to ultrasonic transducers of physiotherapy, in order to determine the sensitivity. One could see that the method implemented here resulted in the coefficients of variation well below the uncertainty described by the standard International Electrotechnical Commission 60866 (IEC, 1987).

The results obtained evaluating two transducers physiotherapy indicated that both exhibit a linear response within the frequency range measured. Any non-linear response could compromise the relationship between the nominal scale of intensity and that actually delivered by the device. Thus, knowledge of the sensitivity adds important information that can be used, for example, by manufacturer, which can optimize the design of equipment from US physiotherapy. 


\section{Introdução}

A onda ultrassônica é uma perturbação mecânica com frequência acima de $20 \mathrm{kHz}$. Em medicina, o ultrassom (US) é aplicado tanto para investigar as propriedades dos tecidos (visando um diagnóstico) como para modificar estas propriedades (terapia). A onda ultrassônica de baixa intensidade tem sido usada com dois propósitos básicos: gerar imagens qualitativas anatômicas (imagens convencionais); e quantificar as propriedades acústicas dos tecidos biológicos como atenuação, velocidade de propagação da onda, retroespalhamento e periodicidade. Já a onda de alta intensidade tem aplicações em terapia, ex. fisioterapia (Fish, 1990), litotripsia (Billard et al., 1990) e hipertermia (Canadian..., 2009).

Os equipamentos de US aplicados à fisioterapia apresentam concepção eletrônica relativamente simples e quase todos possuem o mesmo projeto eletrônico básico. Este consiste, normalmente, de uma fonte de sinal elétrico de alta frequência sintonizada na faixa de 1 a $3 \mathrm{MHz}$, composta de um oscilador e amplificador ligado ao transdutor que converte esses sinais elétricos em energia acústica. Este sinal pode ou não ser modulado de forma a gerar um campo acústico contínuo ou pulsado (Kitchen e Bazin, 1998). Normalmente, o equipamento de ultrassom aplicado à fisioterapia possui um transdutor sem foco, capaz de gerar energia suficiente para produzir alguma alteração biofísica no tecido sem danificá-lo.

A utilização deste recurso, como forma de tratamento, tem o objetivo de estimular o processo natural de cicatrização do tecido, podendo ser aplicado em diferentes tipos de lesões. Desta forma, é um recurso amplamente utilizado em fisioterapia, no tratamento de doenças musculoesqueléticas, particularmente em lesões no tendão, ligamento e bursa sinovial (Speed, 2001). As características das ondas ultrassônicas empregadas em fisioterapia são: frequências de 1 a $3 \mathrm{MHz}$, intensidade entre 0,01 e $3,00 \mathrm{~W} / \mathrm{cm}^{2}$ e modo de operação contínuo ou pulsado.

Não existe concordância na literatura clínica e de pesquisa laboratorial acerca do melhor modo de tratar individualmente cada tipo de lesão (Sá et al., 2006). Um dos motivos principais é a variabilidade biológica (duas lesões do mesmo tipo nunca são idênticas - Kitchen e Bazin, 1998). Por outro lado, os mecanismos de ação e os efeitos terapêuticos gerados pela onda ultrassônica estão diretamente relacionados aos protocolos de tratamento adotados pelo fisioterapeuta, sendo vital sua compreensão. O US usado em tratamento deve oferecer segurança e exatidão, dependendo, portanto, da intensidade, frequência e duração da aplicação (Guirro e Santos, 2002; Ishikawa et al., 2002). Deve-se enfatizar que a calibração do equipamento é um fator primordial para o manuseio de tal recurso no processo de reabilitação. Equipamentos não calibrados quanto à intensidade efetiva gerada podem causar ausência ou diminuição dos benefícios pretendidos ou o agravamento dos sinais e sintomas de doenças pré-existentes, resultando em prejuízo ao paciente e ao próprio terapeuta (Macedo et al., 2003).

Atualmente o método rotineiramente utilizado pelos profissionais para se certificar que os equipamentos estão emitindo radiação ultrassônica é apenas visual. A técnica se baseia na aplicação de uma fina camada de algum meio acoplador (água, gel ou óleo mineral) na superfície do transdutor (popularmente denominado "cabeçote"). Em seguida, com o equipamento em operação, observa-se o aparecimento de bolhas de ar no meio acoplador. Caso isso ocorra, assume-se que o equipamento "está funcionando". Embora tal técnica indique minimamente que o aparelho funciona, além de ser apenas qualitativa, tende a danificar progressivamente $\mathrm{o}$ transdutor, que radia para $\mathrm{o}$ ar.

Guirro e Santos (2002) afirmam que a ausência da calibração da intensidade do aparelho não está relacionada à falta de um procedimento metrológico, mas sim à falta de preocupação dos fisioterapeutas com as condições em que o aparelho está operando (pois não receberam formação para isso) e ao número restrito de parâmetros do equipamento que podem efetivamente ser medido em centros de pesquisa ou fabricantes. Ou seja, a ausência da metrologia como elemento de apoio, integrando o cotidiano dos profissionais, pode reduzir a credibilidade da execução deste recurso terapêutico (Costa-Félix e Azevedo, 2003).

O método utilizado neste estudo para caracterizar os transdutores consiste na calibração por autorreciprocidade, descrito originalmente na norma pela International Electrotechnical Commission 60866 (1987), que tem como objetivo determinar a sensibilidade do transdutor. Esta norma foi cancelada e substituída pela International Electrotechnical Commission 62127-2 (IEC, 2007). Entretanto, a norma de referência deste trabalho será a IEC 60866, pois a versão mais moderna não detalha o procedimento empregado.

A importância de se conhecer a sensibilidade de emissão de um transdutor em geral, e de fisioterapia em particular, reside no fato de que, de posse dessa informação, é possível calcular sua emissão ultrassônica para uma determinada frequência (pressão gerada na sua face) a partir da injeção conhecida de tensão e/ou corrente no seu terminal elétrico. Considerando que se tenha um gerador de sinais eletricamente calibrado (o equipamento de fisioterapia), pode-se inferir o campo 
acústico que o transdutor irá gerar, extraindo-se ou prevendo-se, a partir desse parâmetro, possíveis efeitos biológicos no meio de propagação, uma vez que se utilizem modelos apropriados de comportamento do campo. Em suma, a sensibilidade é um fator de conversão eletroacústico em função da frequência (Lewin et al., 2000).

Os equipamentos de US para fisioterapia têm como principal objetivo gerar aquecimento no meio radiado. Desta forma, o parâmetro mais importante a ser avaliado, na calibração deste equipamento, é a intensidade efetiva entregue ao meio. No entanto, existem outros parâmetros que devem ser analisados para se considerar o equipamento calibrado.

Assim, em resposta à necessidade da realização de um tratamento fisioterapêutico eficaz utilizando o recurso ultrassônico com segurança e exatidão, o objetivo deste estudo é implementar e validar o método de calibração por autorreciprocidade a fim de caracterizar a sensibilidade de transdutores ultrassônicos usados em fisioterapia e evidenciar a contribuição da metrologia como instrumento de apoio à confiabilidade nesse tipo de tecnologia em saúde.

\section{Materiais e Métodos}

Foram utilizados 3 transdutores de US para o experimento. Um deles, aplicado a ensaios não destrutivos (NDT, sigla em inglês) de fabricação da GE Panametrics (General Electric Company, Fairfield, USA), opera na frequência nominal de $2,25 \mathrm{MHz}$ e pertence ao Laboratório de Ultrassom (Labus) da Divisão de Metrologia Acústica e Vibração (Diavi) do Instituto Nacional de Metrologia, Qualidade e Tecnologia (Inmetro). Dois transdutores comerciais usados em fisioterapia, com frequência nominal de operação de $1 \mathrm{MHz}$, pertencentes ao Laboratório de Ultrassom (LUS) do Programa de Engenharia Biomédica (PEB) da COPPE/UFRJ, foram utilizados como equipamentos de teste. Estes transdutores são de fabricação nacional, com tempo de uso variado, da mesma marca, mas modelos diferentes.

Para a realização das medições, também foram utilizados: tanque acústico medindo $25 \times 25 \times 100 \mathrm{~cm}$, com paredes de acrílico e contendo água destilada, alvo refletor de aço, de formato cilíndrico de secção circular com $52 \mathrm{~mm}$ de diâmetro e $78 \mathrm{~mm}$ de comprimento, sistema de posicionamento manual (Newport Corporation, EUA), gerador de funções arbitrárias, com 16 bit $200 \mathrm{MSa} / \mathrm{s}$ [samples per second] AWG (National Instruments, modelo NI PXI-5422), osciloscópio digital (300 MHz $2 \mathrm{GSa} / \mathrm{s}$ [samples per second] - Agilent Technologies, modelo DSO 6032A), resistor de $56 \Omega, 8$ diodos 1 N4148 e termo-higrômetro (Rotronic, modelo HygroPalm). O gerador NI PXI-1042 está integrado com a placa MXI-4 (National Instruments, modelo NI PXI-8331), que faz a comunicação destes com o PC por meio da interface GPIB. Todos os equipamentos pertencem ao Labus/Inmetro, e o sistema, bem como o método utilizado, foram validados conforme Oliveira et al. (2008). A validação foi realizada com transdutores e geradores de sinais distintos dos utilizados naquele estudo, configurando, portanto, distinção clara nas abordagens e desenvolvimento entre os dois trabalhos.

\section{Protocolo de medição}

As medições foram executadas conforme os seguintes passos:

1. Determinar a distância entre o transdutor ultrassônico e o alvo no tanque acústico, que foi feita utilizando uma trena. A distância mínima determinada para todos os experimentos foi de $630 \mathrm{~mm}$.

2. Excitar o transdutor na frequência nominal para encontrar o eco.

3. Maximizar o sinal do eco com a translação do transdutor no plano frontal, transversal e sagital. $\mathrm{O}$ ajuste fino da maximização do eco foi feito atuando-se nos eixos de rotação e inclinação do posicionador manual.

4. Determinar a faixa de frequência a ser medida de acordo com a frequência nominal do transdutor.

5. Ajustar a frequência de repetição para $100 \mathrm{~Hz}$ (com base na frequência de repetição de um equipamento de ultrassom aplicado à fisioterapia).

6. Determinar a faixa de tensão aplicada ao transdutor.

7. Aplicar o primeiro valor de tensão com o primeiro valor de frequência para excitar transdutor.

8. Calcular a sensibilidade utilizando as expressões descritas no método de calibração por autorreciprocidade, conforme apresentado por Abrunhosa (2006), Costa-Félix e Machado (2007) e Oliveira et al. (2008). Para calcular a sensibilidade, é necessário medir a corrente $\left(I_{1}\right)$, a tensão de recepção para circuito aberto $\left(U_{1}\right)$ e estimar o parâmetro de reciprocidade $(J p)$. $\mathrm{Na}$ presente implementação, essas medições são realizadas de forma automática pelo sistema experimental.

9. Coletar cinco vezes o sinal gerado e o eco com os parâmetros determinados no passo 7 (mesma frequência e tensão de alimentação). 
A sensibilidade média é determinada a partir destas cinco medições do sinal gerado e do eco.

10. Repetir o procedimento (passos 8 e 9) com a mesma tensão, modificando a frequência até coletar em toda faixa de frequência selecionada.

11. Mudar a tensão de alimentação e repetir os procedimentos 8 e 9 .

Ao término de todas as medições, obtém-se uma curva de sensibilidade dentro da faixa de frequência escolhida, para cada um dos valores de tensão de alimentação.

\section{Implementação do protocolo}

$\mathrm{O}$ gerador de funções injeta um sinal no transdutor ultrassônico, sem a utilização de um amplificador. Antes de excitar o transdutor, o sinal passa por uma ponte de 8 diodos, sendo 4 montados em um sentido de condução e os outros 4 em paralelo, com sentido de condução contrário (Figura 1). Neste momento é coletado o valor de tensão, após a passagem do diodo $(V c h 0)$. Posteriormente, o sinal passa por um resistor calibrado de $56 \Omega$, onde será coletado o valor de tensão, após a passagem pelo resistor $(V c h 1)$. Ambas as tensões serão utilizadas para calcular o valor da corrente de emissão juntamente com o valor do resistor, por meio da Equação 1:

$I=\frac{V c h 1-V \operatorname{ch} 0}{56 \Omega}$
O sinal alimenta o transdutor que emite uma onda ultrassônica que se propaga no tanque até atingir o alvo. Parte da onda refletida retorna ao transdutor, gerando neste uma tensão correspondente à pressão recebida. Esta tensão $\left(U_{1}\right)$ é medida conforme a Figura 1 e descrito em detalhes em Oliveira et al. (2008) e Abrunhosa (2006).

O cálculo da sensibilidade de transmissão $(S)$ foi adaptado dividindo a Equação 2:

$S=\frac{p_{1}}{I_{1}}$

pela tensão de emissão depois de passar pelo diodo $(V c h 0)$. Esta adaptação implicará o cálculo da sensibilidade dividido pela impedância $(Z)$ e não o valor real de sensibilidade. $O$ valor de sensibilidade será representado em $\mathrm{Pa} / \mathrm{V}$. A Equação 3 detalha o procedimento algébrico para a conversão da grandeza do mensurando:

$S=\frac{p_{1}}{I_{1}} \therefore \frac{S * I_{1}}{V c h 0}=\frac{p_{1}}{V c h 0} \therefore \frac{S}{Z}=\frac{p_{1}}{V c h 0}$

Antes de iniciarem as medições para obtenção da sensibilidade, em cada avaliação o transdutor e o alvo refletor foram colocados no tanque acústico a uma distância mínima de $630 \mathrm{~mm}$ entre eles, com o objetivo de posicionar o alvo no campo distante (zona de Fraunhofer), visando trabalhar com ondas planas. Antes de cada coleta ser iniciada foi realizado um alinhamento entre o transdutor e o alvo, com o

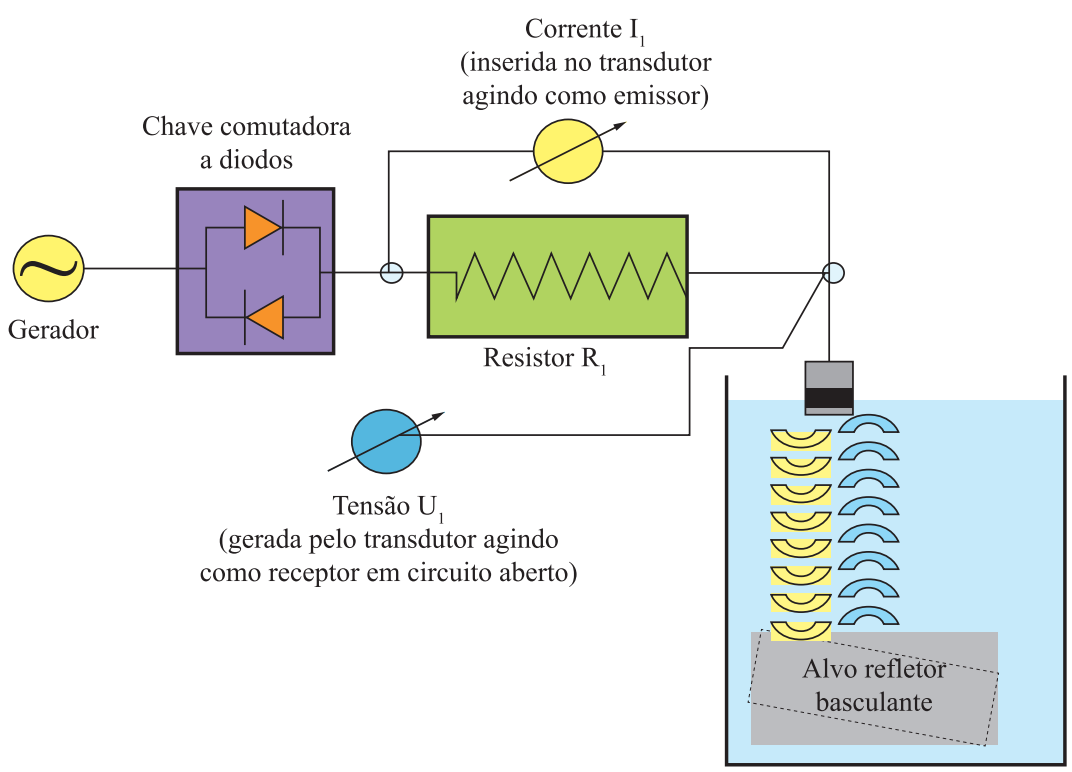

Figura 1. Diagrama de blocos da calibração do transdutor pela técnica de autorreciprocidade extraída de Abrunhosa (2006). Cada diodo do bloco da chave comutadora representa um conjunto de quatro diodos em série.

Figure 1. Diagram for self-reciprocity calibration method, extracted from Abrunhosa (2006). Each diode block change-over switch represents a set of four diodes in series. 
objetivo de maximizar o sinal recebido, com o auxílio do manipulador micrométrico sob o qual estava montado o transdutor. Este manipulador translada o transdutor nos planos frontal, transversal e sagital. Uma vez estabelecida a posição com o sinal maximizado, o transdutor e o alvo refletor são mantidos na mesma posição durante todo o processo de coleta dos dados (Figura 2).

Como sinais de excitação para as calibrações, foram utilizados diversos trens de pulsos, dependendo do transdutor a ser avaliado. Para os transdutores de fisioterapia, com frequência central nominal de $1 \mathrm{MHz}$, variou-se a frequência de 0,9 até $1,15 \mathrm{MHz}$ (em passos de $25 \mathrm{kHz}$, totalizando 11 níveis de frequências) e a tensão nominal de $3,5 \mathrm{~V}$ até $10,5 \mathrm{~V}$, medidos pico a pico (em passos de $1,0 \mathrm{~V}$, totalizando 8 níveis de tensões). Complementarmente, para verificar o sistema, foi repetido o procedimento descrito em Oliveira et al. (2008), utilizando-se um transdutor com frequência central de 2,25 MHz (Panametrics). Foram utilizados diversos trens de pulsos, variando-se as frequências de 1,5 até $3,0 \mathrm{MHz}$, em passos de $150 \mathrm{kHz}$ (totalizando 11 frequências) e 11 valores nominais de tensão (de $3,5 \mathrm{~V}$ até $10,5 \mathrm{~V}$ medidos pico a pico, em passos de $1,0 \mathrm{~V})$. A escolha da frequência de repetição teve como objetivo simular a onda pulsada gerada pelo equipamento de ultrassom para fisioterapia. A maioria dos equipamentos operando em modo pulsado consta de um gerador que emite um trem de pulsos (burst) senoidal de $1 \mathrm{MHz}$ e 30 ciclos (frequência de repetição de $100 \mathrm{~Hz}$ ).

Em todos os casos, foram mantidos fixos a frequência de repetição $(100 \mathrm{~Hz})$ e o número de 30 ciclos por burst. Para a geração do pulso e captação do eco pelo transdutor, foi utilizado um programa em ambiente LabView ${ }^{\circledR}$ para controlar o equipamento NI PXI 1042 (Costa-Félix et al., 2006). Os principais parâmetros da onda a ser gerada eram colocados na tela principal do programa, enquanto na janela intitulada "Reciprocidade" no programa é utilizada para exibir o cálculo da sensibilidade dos transdutores nas frequências e tensões estabelecidas. Ou seja, nesta tela colocam-se os parâmetros das faixas de frequência e de tensão do transdutor (valor inicial, passo e número de pontos desejado). A partir desses valores, o programa cria uma matriz. Cada transdutor foi avaliado 3 vezes em condições de repetitividade a fim de avaliar a exatidão das medições.

O sinal gerado excita o transdutor, que emite um pulso ultrassônico que se propaga pelo tanque acústico até encontrar o alvo refletor. Além de controlar a geração do pulso e a captação do eco pelo transdutor, o programa transfere, processa e armazena os sinais e também monitora a temperatura da água. Estes sinais são gravados em ASCII. O cálculo da sensibilidade

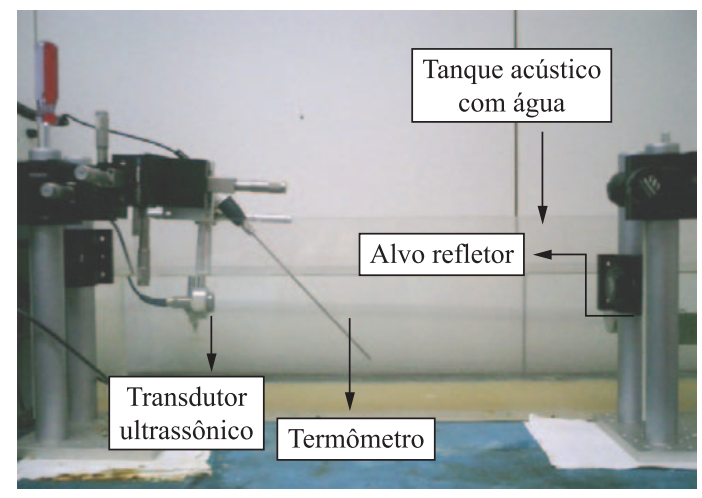

Figura 2. Montagem experimental no tanque acústico.

Figure 2. Experimental setup in the acoustic tank.

do transdutor é determinado com base na norma International Electrotechnical Commission 60866 (IEC, 1997). Com os dados coletados, foram construídos os gráficos de sensibilidade para cada transdutor, o cálculo dos coeficientes de variação e a verificação da linearidade dos transdutores.

\section{Resultados}

Foram avaliados 3 transdutores ultrassônicos. A análise dos dados de sensibilidade dos transdutores teve como objetivo verificar a incerteza do método implementado, a partir do cálculo dos coeficientes de variação, e a linearidade dos transdutores na sua frequência de ressonância com tensão de alimentação de 3,5 a 10,5 Vpp. A Tabela 1 apresenta as especificações dos transdutores ultrassônicos utilizados nos experimentos.

O gráfico da Figura 3 apresenta a curva de sensibilidade de um experimento do transdutor ultrassônico utilizado em NDT (Transdutor 1), nas frequências de calibração utilizadas (faixa 1,5 a 3,0 MHz, passos de 0,15 MHz). Cada curva foi obtida com uma tensão de entrada distinta, conforme descrito na legenda.

O gráfico da Figura 4 apresenta a curva de sensibilidade de um experimento do transdutor de ultrassom utilizado em fisioterapia (Transdutor 2), nas frequências de calibração utilizadas (faixa 0,9 a $1,15 \mathrm{MHz}$, passos de $0,025 \mathrm{MHz}$ ). Cada curva foi obtida com uma tensão de entrada distinta, conforme descrito na legenda.

O gráfico da Figura 5 apresenta a curva de sensibilidade de um experimento com o outro transdutor ultrassônico utilizado em fisioterapia (Transdutor 3), nas frequências de calibração utilizadas (faixa 0,9 a 1,15 MHz, passos de 0,025 MHz). Cada curva foi obtida com uma tensão de entrada distinta, conforme descrito na legenda. 
Tabela 1. Característica de todos os transdutores utilizados nos experimentos.

Table 1. Characteristic of all the transducers used in the experimental.

\begin{tabular}{cccc}
\hline Transdutores & $\begin{array}{c}\text { Frequência nominal } \\
\text { (MHz) }\end{array}$ & $\begin{array}{c}\text { Varredura na frequência } \\
(\mathbf{M H z})\end{array}$ & $\begin{array}{c}\text { Varredura na amplitude } \\
\text { (Vpp) }\end{array}$ \\
\hline 1 & 2,25 & $1,50-3,00$ & $3,5-10,5$ \\
2 & 1,00 & $0,90-1,15$ & $3,5-10,5$ \\
3 & 1,00 & $0,90-1,15$ & $3,5-10,5$ \\
\hline
\end{tabular}
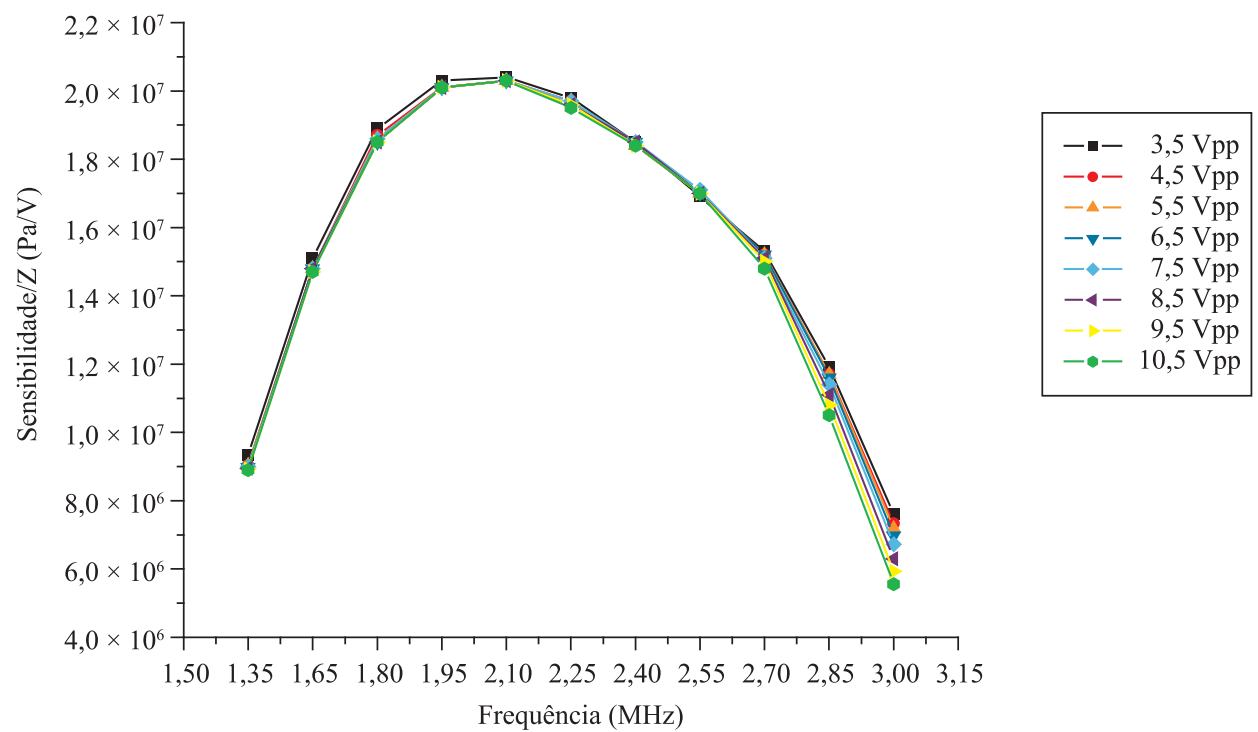

Figura 3. Sensibilidade do Transdutor $1 \mathrm{em}$ função da frequência, para diferentes tensões de alimentação e frequência nominal de $2,25 \mathrm{MHz}$. Figure 3. Sensitivity of Transducer 1 as function of frequency at different voltages with a 2.25 MHz nominal frequency.

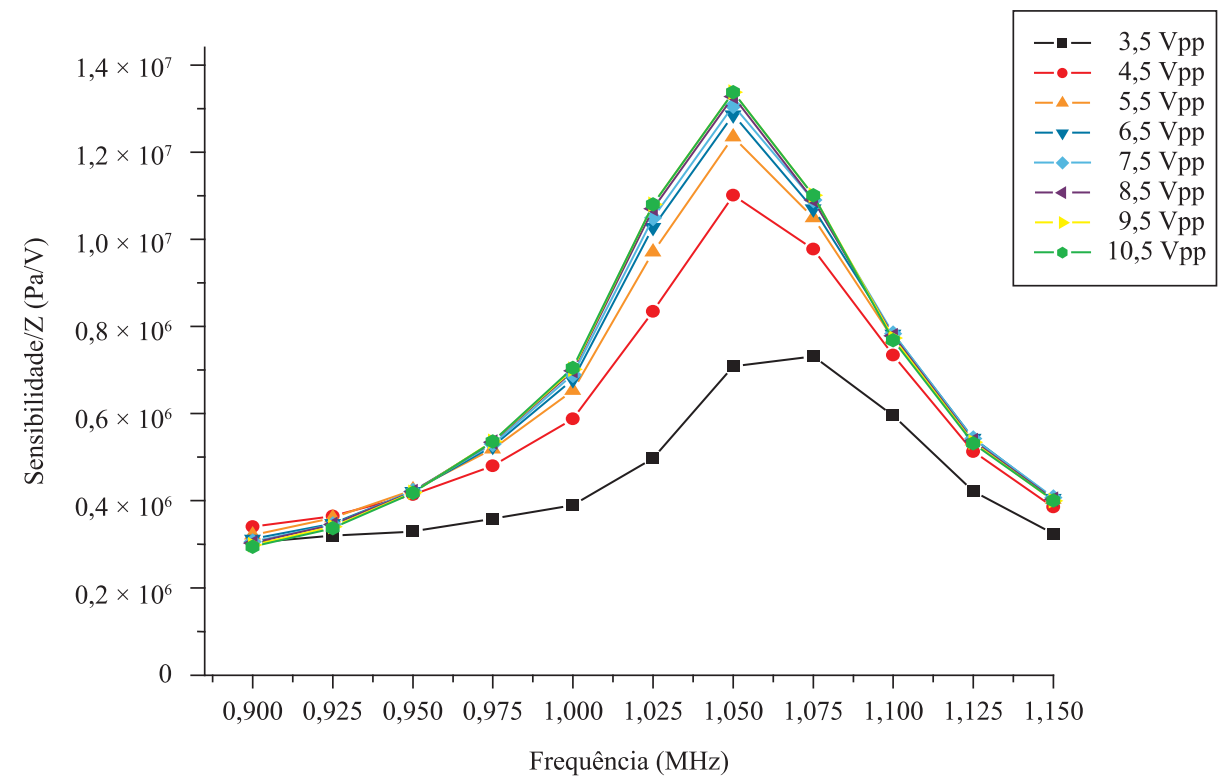

Figura 4. Sensibilidade do Transdutor 2 em função da frequência, para diferentes tensões de alimentação e frequência nominal de $1,00 \mathrm{MHz}$. Figure 4. Sensitivity of Transducer 2 as function of frequency at different voltages with a 1.0 MHz nominal frequency. 


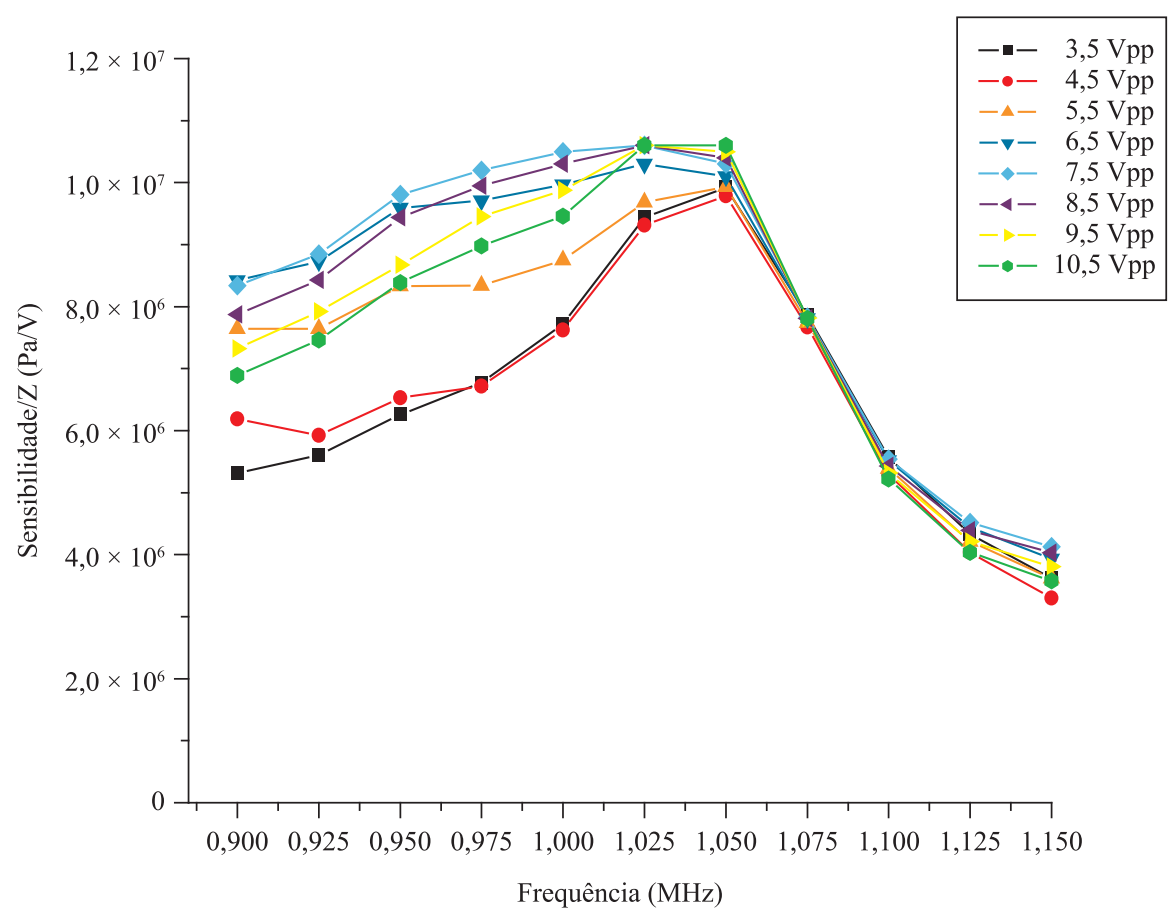

Figura 5. Sensibilidade do Transdutor 3 em função da frequência, para diferentes tensões de alimentação e frequência nominal de 1,00 MHz. Figure 5. Sensitivity of Transducer 3 as function of frequency at different voltages with a $1.0 \mathrm{MHz}$ nominal frequency.

A faixa de frequência utilizada para análise das curvas de calibração dos transdutores foi em torno da frequência nominal de ressonância. Essa é a faixa de melhor resposta do transdutor. Para os transdutores ultrassônico de fisioterapia, essa faixa é mais estreita quando comparada com o transdutor ultrassônico para NDT.

O coeficiente de variação foi utilizado para quantificar a precisão da medição. Ele consiste na divisão do desvio-padrão da sensibilidade dos transdutores pelo valor médio da sensibilidade dos transdutores, nas diferentes tensões de entrada utilizadas. O valor, em porcentagem, deste cálculo também representa a componente Tipo A da incerteza (ou incerteza aleatória) do método implementado. A Figura 6 apresenta as curvas dos valores do coeficiente de variação encontrado nas diferentes tensões de alimentação em cada frequência. Segundo a norma International Electrotechnical Commission 60866 (1987) estes valores, dentro da largura de banda, são menores que a incerteza completa que deve ser menor que $1,5 \mathrm{~dB}$ em nível de sensibilidade de tensão que é vinte vezes o logaritmo na base 10 da razão da sensibilidade do campo livre pela sensibilidade de referência (aproximadamente 18,8\%).

A linearidade (Lin) do transdutor foi calculada conforme descrito pela norma International Electrotechnical Commission 60866 (1987). Esta norma afirma que os valores de sensibilidade medidos para diversas tensões de alimentação devem apresentar variações menores que $10 \%$. Portanto, as Figuras 7 , 8 e 9 apresentam esta variação da sensibilidade de cada transdutor em função da tensão aplicada. A Tabela 2 apresenta os valores de linearidade, em porcentagem, para os transdutores ultrassônicos na faixa de frequência analisada. Linearidade é o valor em porcentagem da diferença do maior valor de sensibilidade pelo menor valor de sensibilidade dividido pelo valor da média da sensibilidade em uma determinada frequência.

\section{Discussão}

Os transdutores de equipamentos comerciais de ultrassom nacionais usados em fisioterapia, geralmente têm somente suas potências verificadas (em fábrica) e sua área de radiação efetiva (ERA - Effective Radiation Area) assumida como sendo a área da cerâmica do transdutor, o que não é tecnicamente correto. Estes transdutores operam em intensidades terapêuticas e, portanto, a segurança biológica torna-se um fator importante a ser considerado. Entretanto, outros parâmetros do transdutor, tais como formato e uniformidade do feixe, sensibilidade, banda de frequência, campo térmico gerado, normalmente não são avaliados por motivos diversos, dentre os 


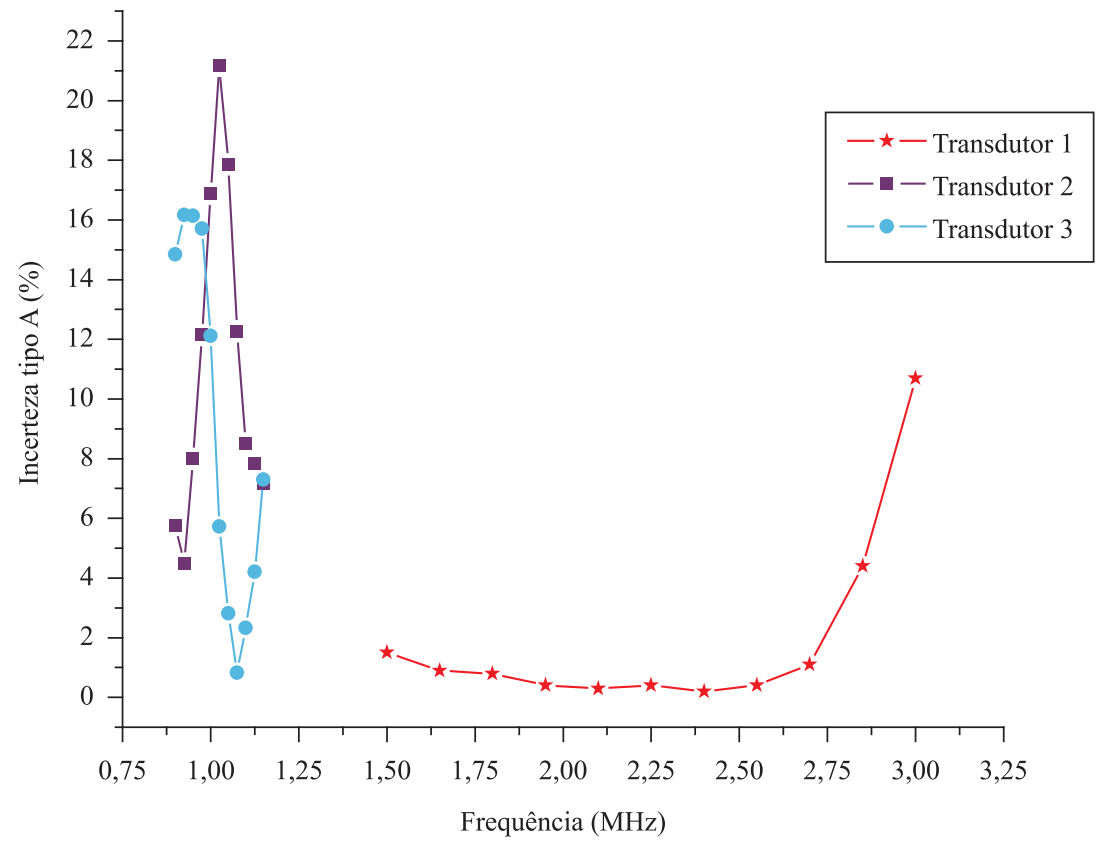

Figura 6. Curva com os valores do coeficiente de variação $(\%)$ do transdutor $1(\star)$, transdutor $2(\bullet)$ e transdutor $3(\bullet)$ em cada frequência com diferentes tensões de alimentação.

Figure 6. Curve with the values of the coefficients of variation (\%) of the transducer $1(\star)$, two transducers $(\square)$ and three transducers $(\bullet)$ at each frequency with different supply voltages.

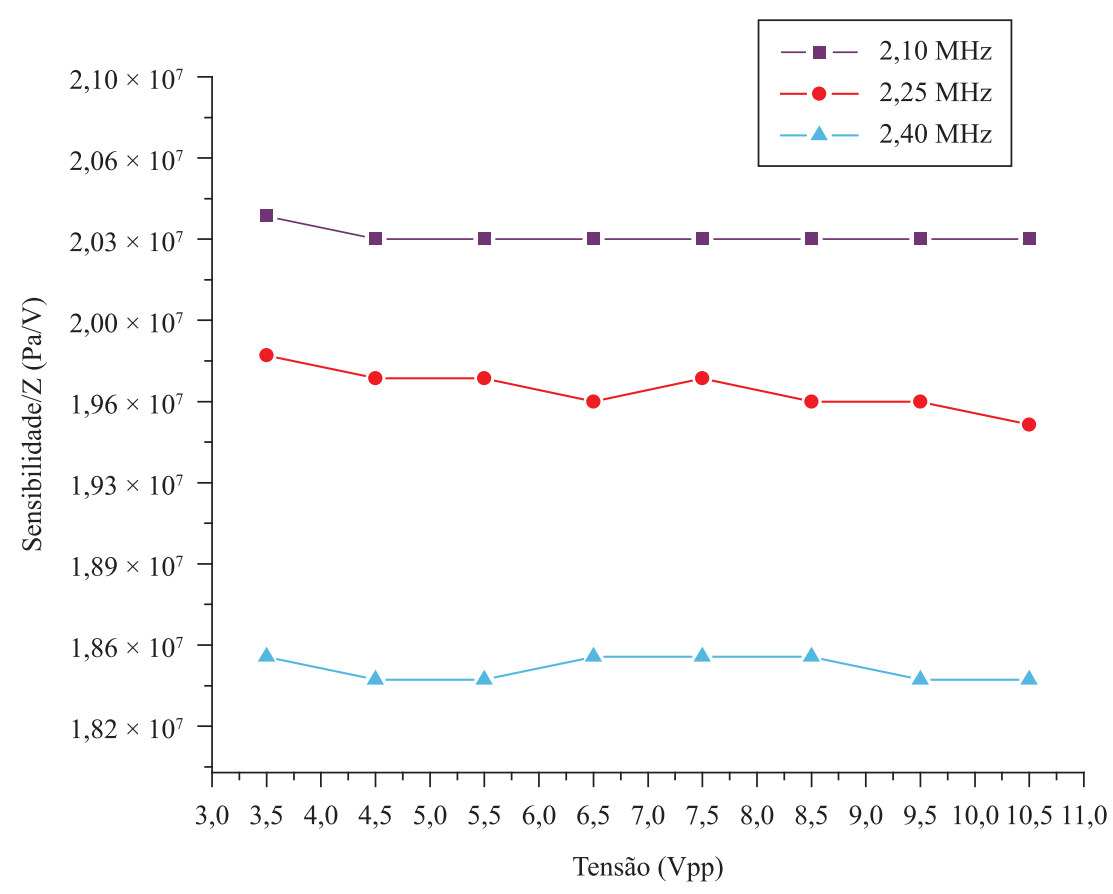

Figura 7. Linearidade do Transdutor 1 através da variação da sensibilidade com o aumento da tensão para três frequências. A curva representada pelo círculo é referente à frequência nominal do transdutor.

Figure 7. Linearity of a Transducer 1 by varying the sensitivity with increasing of voltage with three different frequencies. The curve represented with small circles is referring to the nominal frequency of the transducer. 


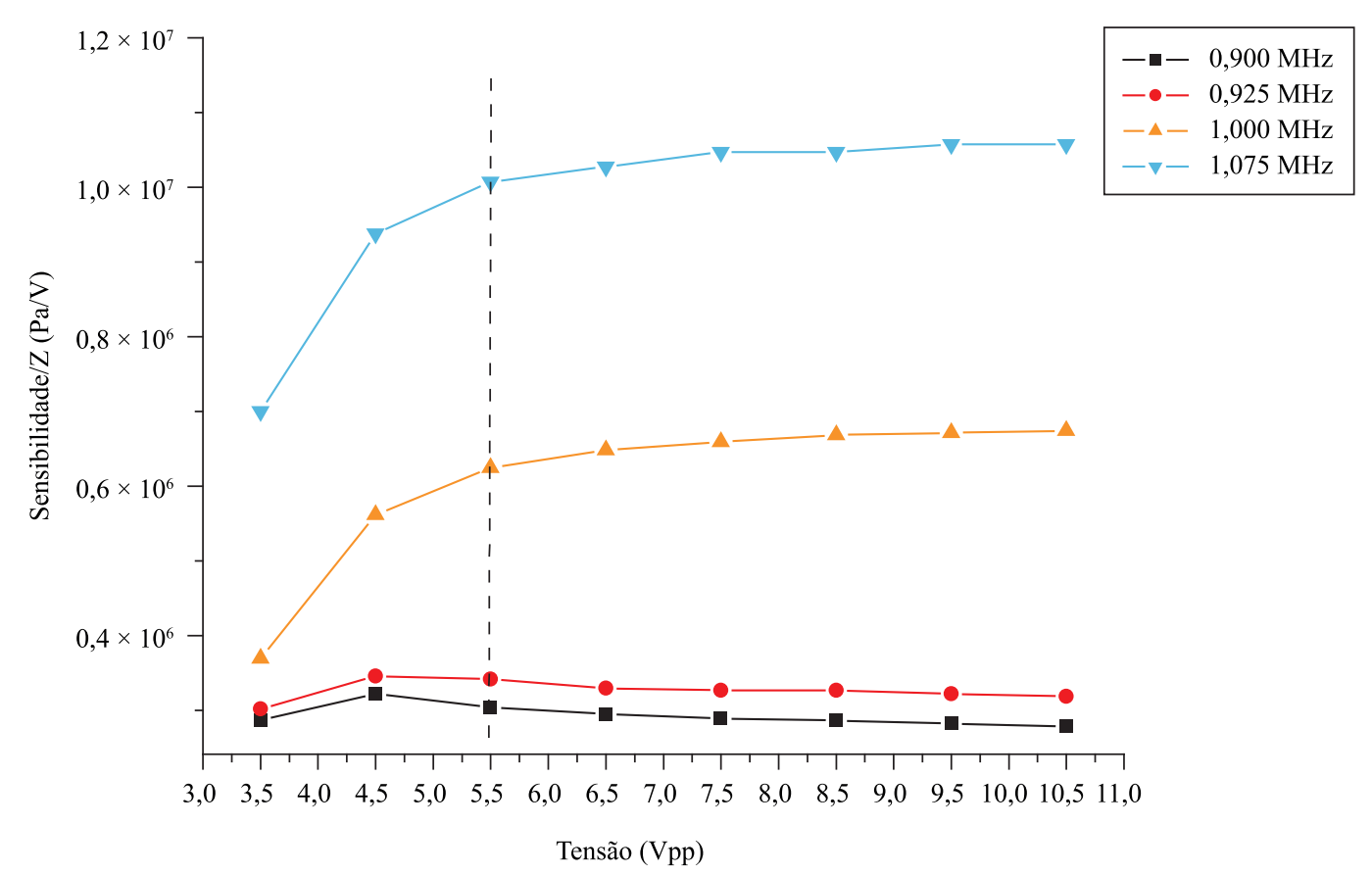

Figura 8. Linearidade do Transdutor 2 através da variação da sensibilidade com o aumento da tensão a frequências diferentes. A curva representada com triângulos não invertidos é referente à frequência nominal do transdutor.

Figure 8. Linearity of a Transducer 2 by varying the sensitivity with increasing of voltage with different frequencies. The curve represented by upright triangles is referring to the nominal frequency of the transducer.

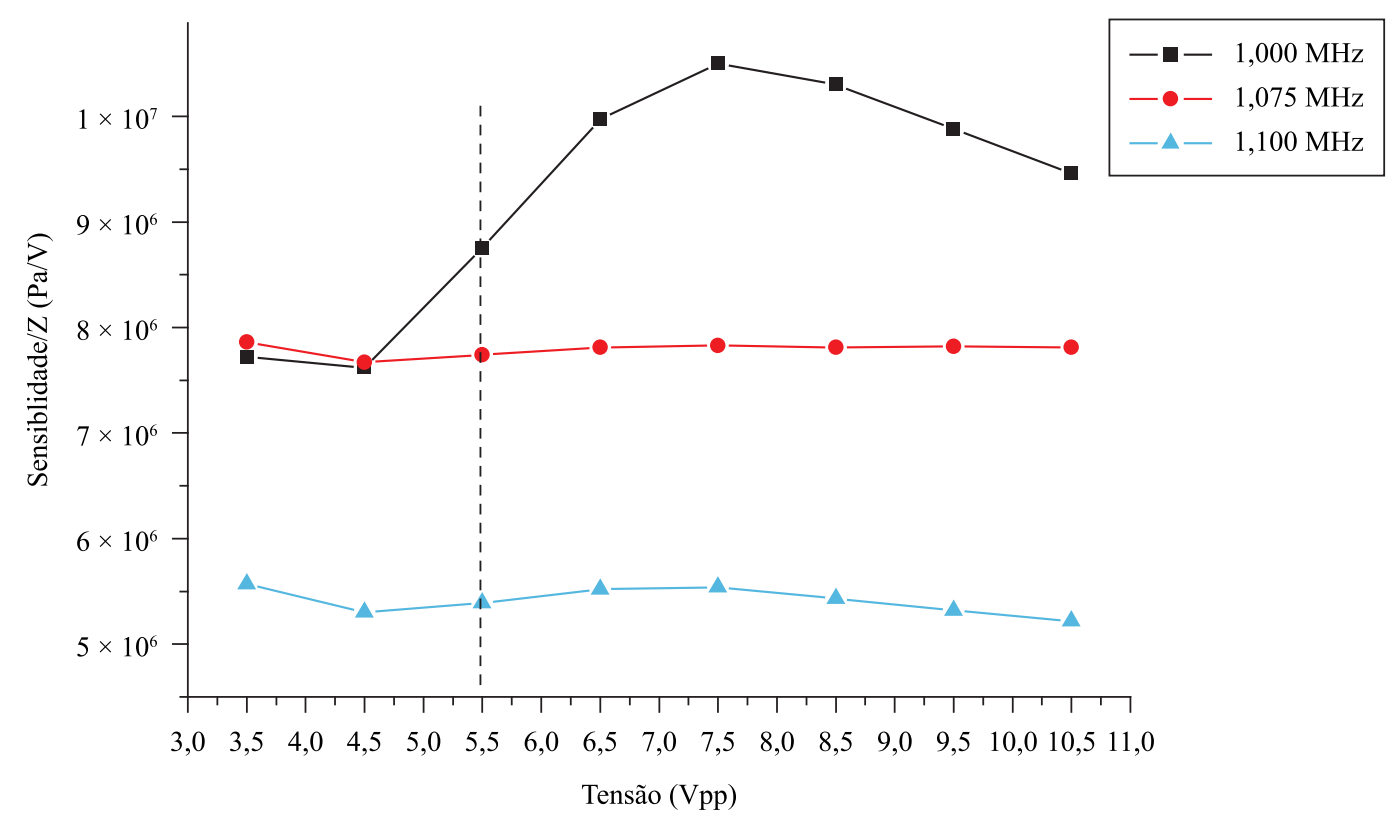

Figura 9. Linearidade do Transdutor 3 através da variação da sensibilidade com o aumento da tensão a frequências diferentes. A curva representada com retângulos é referente à frequência nominal do transdutor.

Figure 9. Linearity of a Transducer 3 by varying the sensitivity with increasing of voltage with different frequencies. The curve represent with small squares is referring to the nominal frequency of the transducer. 
Tabela 2. Valores em porcentagem, representando a linearidade dos transdutores, obtidos pela razão entre a máxima diferença e o valor médio da sensibilidade de uma determinada frequência.

Table 2. Values, in percentage, representing the linearity of the transducers, obtained by the ratio difference between the maximum and the average value of the sensitivity of a certain frequency.

\begin{tabular}{cccc}
\hline Frequência $\mathbf{( M H z )}$ & Transdutor 1 (\%) & Transdutor 2 (\%) & Transdutor 3 (\%) \\
\hline 0,900 & - & 14,46 & 42,79 \\
0,925 & - & 13,11 & 42,87 \\
0,950 & - & 23,08 & 42,45 \\
0,975 & - & 35,19 & 39,92 \\
1,000 & - & 49,41 & 30,63 \\
1,025 & - & 61,52 & 13,14 \\
1,050 & - & 52,02 & 7,58 \\
1,075 & - & 35,96 & 2,39 \\
1,100 & - & 25,16 & 6,44 \\
1,125 & - & 23,24 & 11,26 \\
1,150 & - & 21,16 & 22,08 \\
1,500 & 4,88 & - & - \\
1,650 & 2,97 & - & - \\
1,800 & 2,48 & - & - \\
1,950 & 1,10 & - & - \\
2,100 & 0,77 & - & - \\
2,250 & 1,13 & - & - \\
2,400 & 0,44 & - & - \\
2,550 & 0,85 & - & - \\
2,700 & 3,39 & - & - \\
2,850 & 12,67 & - & - \\
3,000 & 30,62 & - & - \\
\hline
\end{tabular}

quais estão: a falta de infraestrutura laboratorial dos fabricantes; número insuficiente de laboratórios acreditados neste ensaio, e a não exigência deste ensaio por parte da ANVISA. Estes parâmetros têm um papel importante, tanto no desempenho como também na otimização da fabricação dos aparelhos e seus respectivos transdutores.

$\mathrm{Na}$ Figura 6, os valores dos coeficientes de variação para o Transdutor 1 , dentro da faixa de frequência selecionada, foram inferiores a $12 \%$, ou seja, menor que a incerteza máxima descrita na norma International Electrotechnical Commission 60866 (1987) que é de 1,5 dB (aproximadamente 18,8\%). Para os Transdutores 2 e 3 , os valores do coeficientes de variação, dentro da faixa de frequência selecionada de cada transdutor (conforme apresentado na Figura 6) foram inferiores a 21 e 16\%, respectivamente. Esses valores foram encontrados quando o cálculo dos coeficientes de variação foi feito utilizando todas as tensões de alimentação. Porém, quando o cálculo foi realizado desconsiderando os dois primeiros valores de tensão de alimentação, ou seja, 3,5 e 4,5 V medidos pico a pico, os coeficientes de variação encontrados foram inferiores a $3 \%$ para o Transdutor 2 e inferiores a $7 \%$ para o Transdutor 3 , sendo, portanto, menores que a incerteza máxima $(1,5 \mathrm{~dB}$ ou $18,8 \%)$ conforme a norma. Isto ocorre, pois os transdutores para fisioterapia operam em regime de alta potência. Em função da instrumentação utilizada, as amplitudes para as calibrações foram fixadas entre 3,5 e 10,5 V medidos pico a pico, sendo a tensão mínima escolhida em função da ponte de diodos e a tensão máxima em função da limitação do gerador.

Com base nos resultados anteriores, é possível afirmar que a repetitividade do método implementado foi satisfatória. Segundo o Vocabulário Internacional de Metrologia (2008), a repetitividade é o "Grau de concordância entre os resultados de medições sucessivas de um mesmo mensurando efetuadas sob as mesmas condições de medição.”. No entanto, neste trabalho não foi levada em consideração a sua reprodutibilidade que, segundo o VIM, (Vocabulário..., 2008) é o

Grau de concordância entre os resultados das medições de uma mesma grandeza, onde as medições individuais são efetuadas variando-se uma ou mais das seguintes condições: método de medição, observador, instrumento de medida, local, condições de utilização e tempo. Para que uma expressão de reprodutibilidade seja obtida, é necessário especificar as condições que foram alteradas.

O critério de linearidade é descrito pela norma International Electrotechnical Commission 60866 
(1987), a qual afirma que a maior sensibilidade deve diferir menos que $10 \%$ do menor valor de sensibilidade. Assim, o Transdutor 1 (Tabela 2) pode ser considerado linear na frequência nominal de ressonância, utilizando diferentes tensões de entrada. Além disso, pelos gráficos da Figura 7, fica claro que o Transdutor 1 é linear mesmo para as tensões mais baixas, e em toda a faixa utilizada. O mesmo não pode ser dito para os Transdutores 2 e 3 (transdutores de fisioterapia com frequência nominal de $1 \mathrm{MHz}$ ) que, como mostrado na Tabela 2, apresentam valores de linearidade superiores a $10 \%$. No entanto, quando os dois primeiros valores de tensão de alimentação (3,5 e 4,5 V medidos pico a pico) são excluídos, estes transdutores podem ser considerados lineares, na frequência $1,100 \mathrm{MHz}$ para o Transdutor 2 e na frequência de $1,075 \mathrm{MHz}$ para o Transdutor 3. Isto se confirma analisando os gráficos das Figuras 8 e 9 onde, para as tensões mais baixas (3,5 e 4,5 $\mathrm{V}$ medidos pico a pico), as curvas de sensibilidade comprometem a linearidade da frequência nominal (1 MHz para ambos).

Para uma correta expressão dos resultados da calibração, dever-se-ia excluir estas tensões de alimentação, o que influencia não só nos valores dos coeficientes de variação calculados, como também nos valores de linearidade. Como o presente trabalho tem um cunho de desenvolvimento e validação de metodologia, e para melhor ilustrar problemas que podem vir a ocorrer neste tipo de serviço metrológico, nenhum resultado foi omitido e, em consequência, os valores dos coeficientes de variação para os Transdutores 2 e 3 foram maiores que do Transdutor 1 . Vale ressaltar que os transdutores de fisioterapia avaliados são, via de regra, de construção menos rigorosa do que o de NDT, e pode-se supor que respondem melhor apenas para tensões mais elevadas e em faixas mais estreitas de frequência.

\section{Conclusão}

Este trabalho apresenta uma implementação do método de calibração por autorreciprocidade, segundo a norma International Electrotechnical Commission 60866 (1987), aplicado a transdutores ultrassônicos de fisioterapia, com o objetivo de determinar a sua sensibilidade. Pode-se dizer que o método implementado apresentou os valores dos coeficientes de variação bem abaixo da incerteza descrita pela norma International Electrotechnical Commission 60866 (1987). Ou seja, o método está adequado para estudar a sensibilidade de transdutores.

Os resultados obtidos com os 2 transdutores de fisioterapia avaliados indicam que ambos apresentam resposta linear dentro de faixa de frequências avaliada. Isto significa que os transdutores ensaiados possuem uma relação de linearidade entre a tensão de alimentação (excluído as tensões de alimentação de 3,5 e 4,5 V medidos pico a pico) e a sensibilidade calculada. Uma eventual resposta não linear poderia comprometer a relação entre a escala nominal de intensidade e aquela realmente entregue pelo aparelho. Assim, o conhecimento da sensibilidade adiciona informações importantes que podem ser utilizadas, por exemplo, pelo fabricante, que pode otimizar o projeto dos equipamentos de US em fisioterapia. Isto resulta na melhoria da qualidade do produto final e consequentemente, acarreta um tratamento mais eficiente.

Este trabalho mostra resultados da calibração de transdutores de fisioterapia em baixa potência, com um método conhecido da literatura metrológica. Pretende-se dar continuidade a este trabalho buscando formas de caracterizar estes feixes na potência normal de operação, levantando parâmetros que possam ser úteis para a operação segura e eficaz da terapia por US.

\section{Referências}

Abrunhosa VM. Caracterização da sensibilidade de transdutores ultrassônicos de fisioterapia pelo método de autorreciprocidade [dissertação]. Rio de Janeiro: Universidade Federal do Rio de Janeiro; 2006.

Billard BE, Hynynen K, Roemer RB. Effects of physical parameters on high temperature ultrasound hyperthermia. Ultrasound in Medicine \& Biology. 1990; 16(4):409-20. http://dx.doi.org/10.1016/0301-5629(90)90070-S

Canadian Agency for Drugs and Technologies - CADTH In Health Lithotripsy for Kidney Stones or Gallstones: A review of the clinical effectiveness, cost-effectiveness, and guidelines, 2009 [internet]. 2010 Oct [cited 2010 Oct 14]. Available from: http://www.cadth.ca/en/publication/1160

Costa-Félix RPB, Machado JC. Métodos de calibração de hidrofones. Revista Brasileira de Engenharia Biomédica. 2007; 23(3):277-92.

Costa-Félix RPB, Alvarenga AV, Cardona MAR. The brazilian national laboratory of ultrasound. In: IMEKO: Proceedings of the 18th IMEKO World Congress Metrology for a Sustainable Development; 2006 Sep 17-22; Rio de Janeiro, Brazil. Rio de Janeiro; 2006. CD-ROM.

Costa-Félix RPB, Azevedo JA. O panorama nacional e perspectivas futuras em metrologia na área de ultra-som. In: Congresso Brasileiro de Metrologia: Anais do 3th Congresso Brasileiro de Metrologia; 2003 Sep 1-5; Recife, Brazil. Recife; 2003. CD-ROM.

Fish P. Physics and instrumentation of diagnostic medical ultrasound. 2th ed. New York: John Wiley \& Sons; 1990.

Guirro R, Santos SCB. Evaluation of the acoustic intensity of new ultrasound therapy equipment. Ultrasonics. 2002; 39(8):553-7. http://dx.doi.org/10.1016/ S0041-624X(02)00251-2 
ISO-GUM, Guide to expression of uncertainty in measurement, BIPM, IEC, IFCC, ISO, IUPAC, IUPAP, OIML, Geneva, Switzarland, 2008. Available from: www.bipm.org/en/publications/guides/gum.html.

International Electrotechnical Commission - IEC. 60866:1987 Characteristics and calibration of hydrophones for operation in the frequency range $0.5 \mathrm{MHz}$ to $15 \mathrm{MHz}$. Geneva: IEC; 1987. International Electrotechnical Commission - IEC. 621272:2007, Ultrasonics - Hydrophones - Part 2: Calibration for ultrasonic fields up to $40 \mathrm{MHz}$. Geneva: IEC; 2007.

Ishikawa NM, Paes LFC, Alvarenga AV. Análise do desempenho de equipamentos de ultra-som para fisioterapia, operando na cidade do Rio de Janeiro, conforme a norma NBR/IEC 1689 da ABNT. Revista Brasileira de Fisioterapia. 2002; 6(2):63-9.

Kitchen S, Bazin S. Eletroterapia de Clayton. 11th ed. Barueri: Manole; 1998.

Lewin PA, Lypacewicz G, Bautista R, Devaraju V. Sensitivity of ultrasonic hydrophone probes below $1 \mathrm{MHz}$. Ultrasonics. 2000; 38(1-8):135-9.
Macedo AR, Alvarenga AV, Pereira WCA. Mapeamento de feixe ultra-sônico utilizando as propriedades cromotérmicas dos cristais líquidos colestéricos. Revista Brasileira de Engenharia Biomédica. 2003; 19(2):61-38.

Oliveira EG, Costa-Félix RPB, Alves CHF. Validação de um sistema de calibração de transdutores de ultra-som por auto-reciprocidade. Revista Brasileira de Engenharia Biomédica. 2008; 24(1):123-30.

Sá JNB, BUNN PS, Pereira WCA. Análise dos fundamentos das dosagens indicadas para a aplicação do ultra-som em fisioterapia, In: CBEB: Anais do 20th Congresso Brasileiro de Engenharia Biomédica; 2006 Out 22-26; São Pedro, Brazil. São Pedro; 2006. p. 815-8.

Speed CA. Therapeutic ultrasound in soft tissue lesions. Rheumatology. 2001; 40(12):1331-6. PMid:11752501. http://dx.doi.org/10.1093/rheumatology/40.12.1331

Vocabulário Internacional de Metrologia - VIM. Conceitos fundamentais e gerais e termos associados. VIM; 2008. Tradução autorizada pelo BIPM da 3th edição internacional do VIM - International Vocabulary of Metrology - Basic and general concepts and associated terms - JCGM 200:2008.

\section{Autores}

Viviane Mendes Abrunhosa, Rodrigo Pereira Barretto da Costa-Félix

Laboratório de Ultrassom, Diretoria de Metrologia Científica e Industrial, Divisão de Metrologia Acústica e de Vibrações, Instituto Nacional de Metrologia, Qualidade e Tecnologia - Inmetro, CEP 25250-020, Duque de Caxias, RJ, Brasil 